\title{
Neutrinos from WIMP annihilations in the Sun including neutrino oscillations $\ddagger$
}

\author{
Mattias Blennow $^{1}$, Joakim Edsjö ${ }^{2}$ and Tommy Ohlsson ${ }^{1}$ \\ ${ }^{1}$ Department of Theoretical Physics, School of Engineering Sciences, Royal Institute \\ of Technology (KTH) - AlbaNova University Center, SE-106 91 Stockholm, Sweden \\ 2 Department of Physics, Stockholm University - AlbaNova University Center, \\ SE-106 91 Stockholm, Sweden \\ E-mail: emb@kth.se, edsjo@physto.se, tommy@theophys.kth.se
}

\begin{abstract}
The prospects to detect neutrinos from the Sun arising from dark matter annihilations in the core of the Sun are reviewed. Emphasis is placed on new work investigating the effects of neutrino oscillations on the expected neutrino fluxes.
\end{abstract}

PACS numbers: 95.35.+d, 14.60.Pq

Submitted to: Phys. Scr.

‡ Presented by J. Edsjö. 

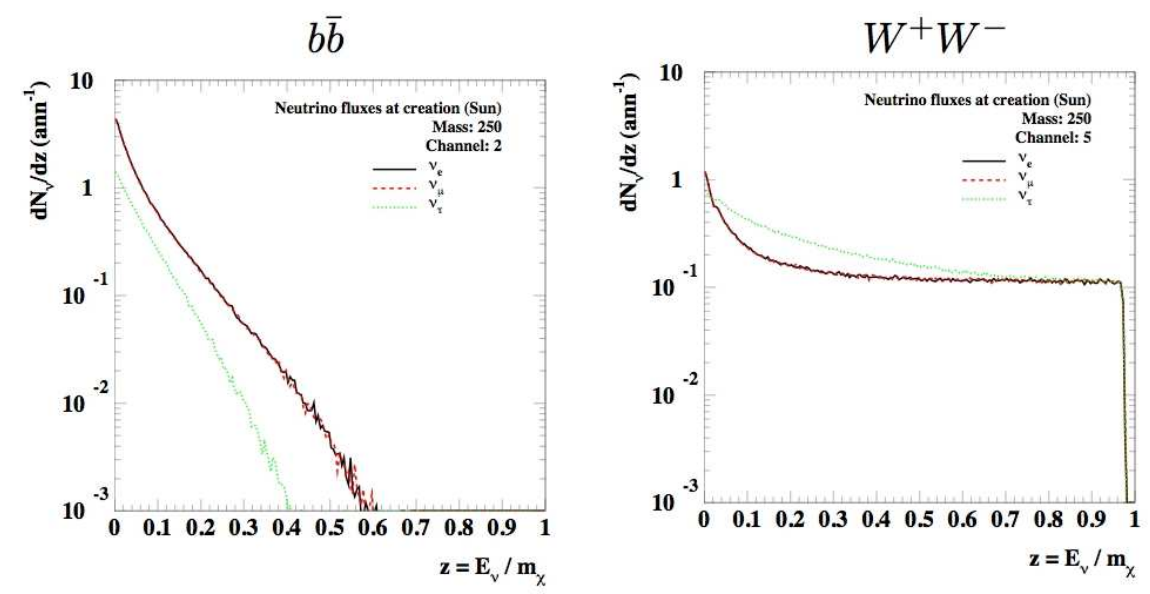

Figure 1. Examples of WIMP annihilation spectra.

\section{Introduction}

From cosmological and astronomical observations, it has been shown that only $4 \%$ of our Universe is consisting of ordinary baryonic matter, $22 \%$ of dark matter, and the remaining $74 \%$ of dark energy. One of the main dark matter candidates are the socalled Weakly Interacting Massive Particles (WIMPs). In order to search for WIMPs, one can either make efforts to detect them directly through their interactions with matter, or do indirect searches for e.g. neutrinos arising from WIMP annihilations in the Sun and in the Earth. Here we will only study the prospect of indirect searches for WIMPs annihilating in the center of the Sun, where our focus is mainly to present a full event-based Monte Carlo simulation for the neutrino fluxes at the Earth.

\section{WIMP capture and annihilation in the Sun}

WIMPs in the Milky Way halo can scatter in the Sun and be gravitationally bound to it. Eventually, they will scatter again and sink to the core of the Sun. In the core, WIMPs will accumulate and can annihilate and produce neutrinos. In Fig. 1, we present two examples of WIMP annihilation spectra, one from the $b \bar{b}$ channel and another from the $W^{+} W^{-}$channel. As all figures in this text, Fig. 1 has been produced for a WIMP mass of $m_{\chi}=250 \mathrm{GeV}$. Note that these annihilation spectra show the initial fluxes at the center of the Sun, and therefore, there are no effects from neutrino oscillations.

\section{Neutrino interactions}

On the way out of the Sun, neutrinos can participate in both charged- and neutralcurrent interactions. Neutral-currents degrade the energy of the neutrinos, whereas charged-currents give a charged lepton, which means that electrons and muons are 


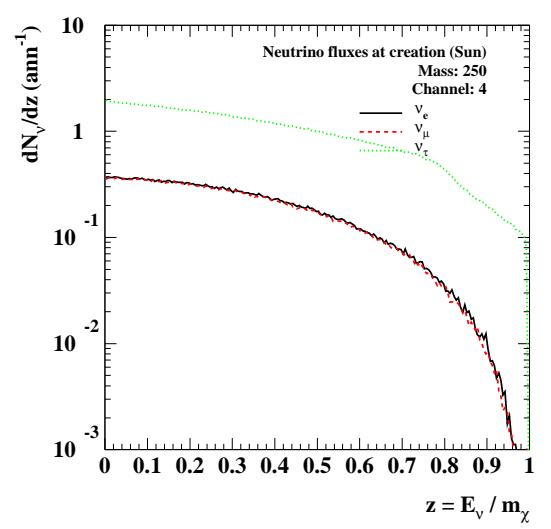

Figure 2. Initial neutrino fluxes at the center of the Sun.

stopped before they can give neutrinos, but tau leptons will decay and produce new neutrinos (regeneration).

Then, what about the spectra beyond the Sun? At the surface of the Sun, some of the neutrinos have interacted, and thus, degrading the flux at high energies. However, some of these neutrinos reappear at low energies both from neutral-current interactions and tau decays.

\section{Neutrino oscillations}

We use a completely general three-flavor neutrino oscillation scheme (with matter effects included) and a realistic solar model [1]. Thus, at the surface of the Sun, we obtain the fluxes in a general format (including both amplitudes and phases of the neutrino oscillations). Furthermore, in our computations, neutrino oscillations and interactions are treated simultaneously. We have used the following values of standard neutrino oscillation parameters (which are the central values from Ref. [2] with no CP violation in neutrino oscillations and a normal neutrino mass hierarchy):

$$
\begin{array}{ll}
\theta_{12} & =33.2^{\circ} \\
\theta_{13} & =0 \\
\theta_{23} & =45.0^{\circ} \\
\delta & =0 \\
\Delta m_{21}^{2} & =8.1 \cdot 10^{-5} \mathrm{eV}^{2} \\
\Delta m_{31}^{2} & =2.2 \cdot 10^{-3} \mathrm{eV}^{2} .
\end{array}
$$

In Fig. 2, we plot the initial neutrino fluxes at the center of the Sun, i.e., at the point of production, assuming annihilation to $\tau^{-} \tau^{+}$with the mass of the WIMPs being $m_{\chi}=250 \mathrm{GeV}$. Next, in Fig. 3, we show how the spectra are modified after the neutrinos have propagated through the interior of the Sun, i.e., how the spectra look on the distance of one solar radius from the center of the Sun. 


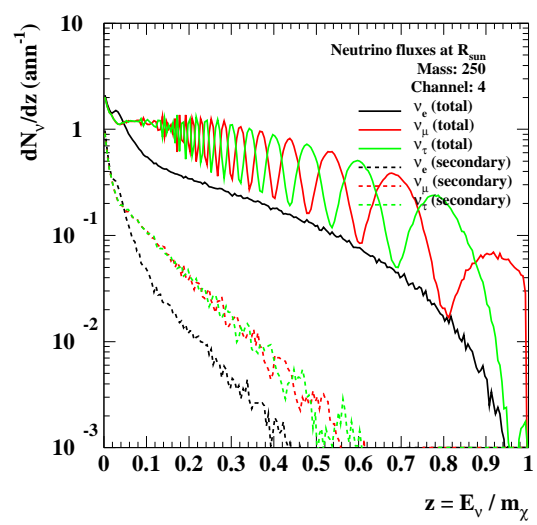

Figure 3. Neutrino fluxes at the surface of the Sun.

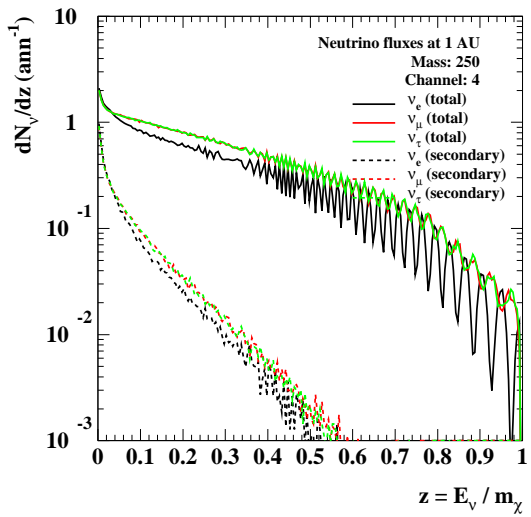

Figure 4. Neutrino fluxes at $1 \mathrm{AU}$.

\section{Propagation to and in the Earth}

First, for the propagation to the Earth, vacuum neutrino oscillations to the Earth are included in the same three-flavor neutrino setup. In addition, effects of the eccentricity of the Earth's orbit are included. In Fig. 4, we show an example of the propagation of neutrinos from the surface of the Sun to the distance of the Earth, i.e., on the distance of 1 AU).

Second, for the propagation in the Earth, matter effects are included in the neutrino oscillations as well. Our simulations are made with a time stamp to include effects of the Earth's distance to the Sun (due to the eccentricity of the orbit) and rotation (affects the distance traversed in the Earth). For illustration, we pick a detector at latitude $-90^{\circ}$ (which could be the IceCube neutrino telescope) and average over one year. The result is presented in Fig. 5, which shows the effect of the Earth's orbit. 


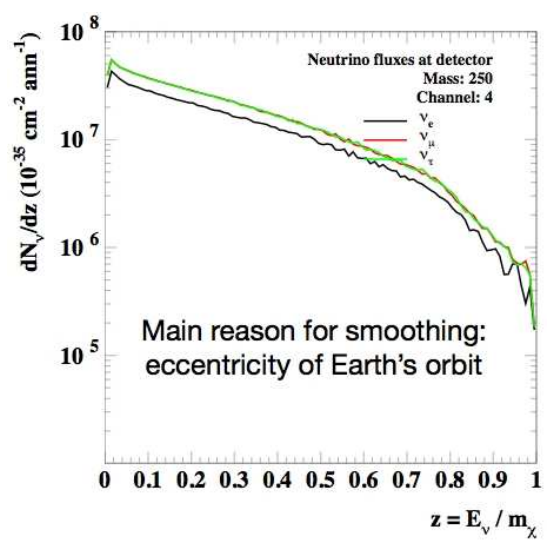

Figure 5. Final neutrino spectra at the detector.

\section{Summary and conclusions}

For typical WIMP masses (i.e., $10 \mathrm{GeV}-10^{5} \mathrm{GeV}$ ), neutrino oscillations effectively:

- average $\nu_{\mu}$ and $\nu_{\tau}$ on the way out of the Sun,

- average $\nu_{\mathrm{e}}$ and $\nu_{\mu} / \nu_{\tau}$ on the way to the Earth, and

- wash out the remaining oscillation patterns in the spectra due to the eccentricity of the Earth's orbit.

Note that the full scheme described above is implemented as a complete eventbased Monte Carlo code. In addition, it should be mentioned that other computations of neutrinos from WIMP annihilations have been performed, such as the study by Cirelli et al. [3]. However, their results are not event-based.

\section{Acknowledgments}

This work was supported by the Royal Swedish Academy of Sciences (KVA) and the Swedish Research Council (Vetenskapsrådet), Contract No. 621-2002-3577, 621-20036025, 621-2005-3588.

\section{References}

[1] J. N. Bahcall, A. M. Serenelli and S. Basu, "New solar opacities, abundances, helioseismology, and neutrino fluxes," Astrophys. J. 621 (2005) L85 [arXiv:astro-ph/0412440].

[2] M. Maltoni, T. Schwetz, M. A. Tortola and J. W. F. Valle, "Status of global fits to neutrino oscillations," New J. Phys. 6 (2004) 122 [arXiv:hep-ph/0405172].

[3] M. Cirelli, N. Fornengo, T. Montaruli, I. Sokalski, A. Strumia and F. Vissani, "Spectra of neutrinos from dark matter annihilations," Nucl. Phys. B 727 (2005) 99 [arXiv:hep-ph/0506298]. 